\title{
Impact of intra-flow network coding on the relay channel performance: an analytical study
}

\author{
Anya Apavatjrut*, Claire Goursaud ${ }^{\dagger}$, Katia Jaffrès-Runser ${ }^{\ddagger}$ and Jean-Marie Gorce ${ }^{\dagger}$ \\ *Computer Engineering, Chiang Mai University, Chiang Mai, Thailand \\ Email: anya@eng.cmu.ac.th \\ †Universite de Lyon, INRIA / INSA-Lyon, CITI, Lyon, France \\ Email: firstname.lastname@insa-lyon.fr \\ ‡University of Toulouse IRIT-INPT ENSEEIHT, Toulouse, France \\ Email: katia.jaffres-runser@irit.fr
}

\begin{abstract}
One of the most powerful ways to achieve transmission reliability over wireless links is to employ efficient coding techniques. This paper investigates the performance of a transmission over a relay channel where information is protected by two layers of coding. In the first layer, transmission reliability is ensured by fountain coding at the source. The second layer incorporates network coding at the relay node. Thus, fountain coded packets are re-encoded at the relay in order to increase packet diversity and reduce energy consumption. Performance of the transmission is measured by the total number of transmissions needed until the message is successfully decoded at the destination. We show through both analytical derivations and simulations that adding network coding capabilities at the relay optimizes system resource consumption. When the source uses a random linear fountain code, the proposed two-layer encoding becomes more powerful as it reduces the transmission rate over the direct link between the source and the destination.

Index Terms-Intra-flow network coding, random linear foun-
\end{abstract} tain codes, relay channel, cooperative communications

\section{INTRODUCTION}

Maximizing the reliability of wireless transmissions has triggered extensive studies in the last decade. This is linked to the lossy nature of wireless transmissions which originate in various phenomenons such as fading, interferences, etc.

For a direct transmission link, reliability is usually guaranteed by leveraging coding techniques for the design of efficient link layer protocols. Such protocols verify data integrity and request its retransmission to recover possible losses on the channel. They derive from traditional Automatic RepeatreQuest protocols(ARQ, HARQ) [1]. The considered codes, called Forward Error Correcting (FEC) codes, are erasure codes applied by the source of the original data. They ensure that the destination will be able to recover any lost pieces of information and thus receives the integrality of the original data. Based on the encoding/decoding technique, FEC codes can be categorized as: RS (Reed Salomon) [2], LDPC (Low Density Parity Check) [3], RLF (Random Linear Fountain) [4], LT (Luby Transform) [5], Raptor [6], etc. Thanks to these codes, transmission errors can be overcome with the help of additional amount of redundancy introduced by the encoding process.

More recently, in a network where information packets are to be transferred through a certain number of nodes, cooperation among them has been shown to clearly increase transmission performance [7]. In a cooperative transmission scheme, a packet is to be transferred from a source to a destination with the help of a certain number of relay nodes. Cooperation can be even more beneficial when the direct link between the source and the destination is poor. With cooperation, spatial diversity of relays can be leveraged: information may travel through multiple paths in parallel to the destination, each one of them experiencing different channel conditions. Although multipath transmissions increase spatial diversity, it may introduce unnecessary redundancy. This is typically the case when the destination receives several copies of the exact same packet.

In order to optimize the amount of redundancy in a system due to both coding and/or cooperation, we have introduced in [8] a lightweight network coding solution aiming at optimizing system resource consumption. Our goal is to create a solution that both achieves perfect reliability and minimizes the number of encoded packet transmissions. Based on the presented simulation results, we have validated that network coding necessitates a reduced number of transmissions in a cooperative network by introducing packet diversity in the system.

The context of this study is to analytically investigate the efficiency of intra-flow network coding solutions for single relay cooperative communications. We consider here a FountainCoding-and-Forward communication scheme [9] where the relay forwards a newly encoded packet following a simple and lightweight intra-flow network code. No prior decoding precedes the network coding stage, only the destination recovers the original data. Reliability is ensured using a random linear fountain code at the source. As such, a single packet acknowledges that the original data has been properly decoded at the destination and that the source can stop the fountain of encoded packets.

We propose in this paper to characterize the efficiency of the communication by deriving the equivalent end-toend probability of success which characterizes the reliability of the complete relay network assuming simple intra-flow network codes. From this end-to-end probability of success, it is straightforward to derive the average number of encoded 
packet transmissions before original data is decoded. The average number of encoded packets is directly proportional to the transmission delay and the energy consumption of the network. Next, we introduce a variable, the transmission rate, that captures the decision of the relay to fountain-codeand-forward a received packet or not. Optimal decision is derived to minimize the average number of encoded packets. At optimal transmission rate, we show that the number of transmitted packets is reduced with the intra-flow network code at the relay. However, the gain is relatively small. We exhibit in the last part that the full capability of network coding is obtained as the source transmits a random linear fountain code. Indeed, the XOR combination of RLF packets create valid codewords, resulting in faster decoding at the destination.

Complementary yet different studies to this work can be found in [9] and [10]. Qin et al. [9] focus on deriving a capacity bound for the single relay cooperative channel while Kurniawan et al. propose a combined fountain and network coding solution for a 2-relay cooperative channel. The two relays are trying to decode in parallel a message encoded with a RLF code. As soon as one of them has recovered the original data, the source stops transmitting and the relay with complete information creates a new stream of RLF codes. In our approach, we aim at not decoding the whole stream of RLF codes at the relays in order to keep energy and computation at a low level.

The paper is organized as follows. Preliminaries are given in Section II and our system model is defined in Section III. The core of the end-to-end probability of success derivation is given in Section IV and the derivation of the optimal transmission rate in Section V. Section VI studies the impact of the overhead introduced by fountain codes in the transmission and finally, Section VII concludes the paper.

\section{PRELIMINARIES}

The aim of this section is to give an overview of the building blocks of our 2-layer coding cooperative communication scheme. Here, we give an overview of fountain codes, cooperative communications and network coding.

\section{A. Fountain codes}

Fountain codes belong to a category of erasure codes that are rateless and universal. The rateless and universal property can be explained by the fact that when a source transmits an endless sequence of encoded information to the destination, the transmission is halted once the destination receives a sufficient amount of data to successfully decode the original information. In this kind of transmission, the rate of the code is adapted onthe-fly depending on the channel quality. As a result, despite the error probability of the channel, fountain codes are usually performing well and adapt their rate to be optimal over any kinds of channel [4], [11].

There exist several types of fountain codes based on the encoding/decoding process: Random Linear Fountain codes (RLF) [4], Luby Transform code (LT) [5], Raptor code [6], etc. For the RLF, the source randomly selects data packet with equal probability to create a sequence of encoded packet before transmitting them to the destination. The destination later decodes these packets with Maximum Likelihood technique. It has been shown that, when compared to the others, the RLF code provides the minimum coding overhead but at the cost of a higher decoding complexity. Besides, the RLF are more XOR-friendly, as the combination of two RLF codeword flow leads to a RLF flow.

Thus, in this work, we consider that the destination can handle any decoding, and we will focus on the RLF as they are more suitable for network coding.

\section{B. Cooperative Communications}

In a large-scale network where a system is composed of several nodes, transmission performance can be enhanced with the help of several relays. With cooperative communication, relay nodes collaborate in relaying information from the source to the destination. Transmission through several relays propagates through multiple paths and introduces diversity called cooperative diversity or multi-users diversity. Achieving cooperative diversity can be obtained through different means from various levels of communications as following.

At signal level, cooperative diversity is analog to the diversity obtained in a system with multiple antennas [7], [12]. These diversity can be obtained when relay nodes in the system relay their information thanks to several relaying technique such as amplify-and-forward, decode-and-forward, etc. These techniques help reducing the total number of transmissions as well as increasing the spectral efficiency of the transmission. Recently, relaying with fountain codes called fountain codingand-forward has been demonstrated to outperform other existing relaying strategies [9].

At packet level, cooperative communication can be seen as an opportunistic routing protocol where relay nodes participate in relaying information from one node to another node .

\section{Network Coding}

Network coding has been proposed by Ahlswedes and al. to improve transmission rate in multicast scenario [13]. This technique can help improving the system's capacity to approach the min-cut capacity which is the maximum capacity of the system. The study in [14] affirms that the optimum capacity can be reached when a relay node re-encodes and/or decodes the information by transmitting a combination in $\mathbb{F}_{q}$ where $q \in \mathbb{N}$. The benefits of network coding is that it can increase transmission rate, scalability, complexity, delay, etc.

\section{SYSTEM MODEL}

We are interested in the simplest cooperative scheme called a relay network. A relay network is composed of three nodes: source (S), destination (D) and a relay (R) as shown in figure 1. The source disposes of a certain amount of information to be transmitted to the destination. This information is transmitted directly and through a relay node who assists in the transmission. We characterize the link quality based on a link 
probability $P$. The link probability between S-D, S-R and RD is represented by $P_{S D}, P_{S R}$ and $P_{R D}$ respectively. We suppose that each sensor disposes of only one antenna and that the transmission is half-duplex.

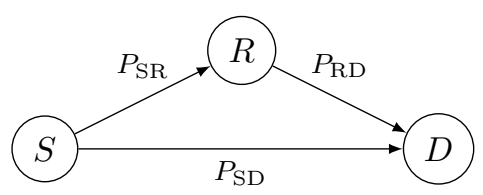

Fig. 1. Relay Channel

We call "round of transmission" the complete transmission stage of a data packet from the source to the destination. Each round is composed of two steps executed in two consecutive time slots. In the first time slot, the source broadcasts its information. Both the destination and the relay can receive the packet in this first slot. In the second time slot, the relay broadcasts its previously received information to the destination. The source is silent. We suppose that this timemultiplexed scheme is perfect in the sense that the emitting node is never interfered by the other one.

\section{END-TO-END SUCCESS PROBABILITY}

In the remaining of the paper, successful transmission refers to the case where a given packet is received during the reception. We derive the end-to-end reception success probability for different network coding scenarios at the relay nodes.

\section{A. Classical relaying}

For the simplest cooperative scheme, the relay simply forwards the packet. There is no network coding involved. The end-to-end success probability of the system can be computed as the probability that the packet is received successfully via the direct link S-D, or by the relay link S-R followed by R-D. Formally:

$$
P=P_{S D}+\left(1-P_{S D}\right)\left(P_{S R} P_{R D}\right)
$$

It is straightforward to derive the average number of transmissions needed in an ARQ protocol using $N_{t x}=\left(1+P_{\mathrm{SR}}\right) / P$.

We can intuitively expect the success probability to increase when the relay node is allowed to process the information through efficient re-encoding at the relay node. In the rest of the section, we focus on how to increase transmission performance in terms of the number of transmissions using a lightweight network coding protocol applied at the relay. To do so, we are interested in deriving a theoretical analysis of the success probability when network coding is present at the relay. To simplify our study, we suppose in the first place that no coding is applied at the source. Consider that a source transmits a sequence of packets $P=\left\{p_{1}, p_{2}, \ldots, p_{\infty}\right\}$ to $\mathrm{D}$. We are interested in this section to derive the end-toend probability of success over a relay channel as a function of the link probability between the nodes and of the relaying technique.

\section{B. Preliminary study : $P_{S R}=1$}

To lessen the analysis complexity, we first present the case where the link probability between the source and the relay $\mathrm{S}-\mathrm{R}$ is supposed to be perfect $\left(P_{S R}=1\right)$.

Furthermore, we consider that the relay combines the received packets as follows:

Scenario 1 : relay node applies network coding to any two consecutive packets from the source point of view.

In this scenario, any packet sent by the source is tagged with a specific sequence number. The relay stores the received packets in its buffer. For each received packet with a sequence number $i$, the relay node searches in its buffer if there exists a previously received packet with the previous sequence number $i-1$. If it exists, the relay node transmits a combination in $\mathbb{F}_{2}$ of $p_{i} \oplus p_{i-1}$, otherwise, the relay solely transmits packet $p_{i}$. This relaying process is described in Algorithm 1 .

For $P_{S R}=1$, each packet sent by the source is received by the relay. Thus, the relay always transmits $p_{i} \oplus p_{i-1}$ towards the destination.

We note that the successful transmission (and/or decoding) of a packet $p_{i}$ can be obtained with three different cases:

- In the first case, $p_{i}$ is received successfully at the destination through the direct link S-D. Its probability of success is $P_{S D}$

- In the second case, $p_{i}$ can be decoded from a successfully received encoded packet which contains a combination of $p_{i} \oplus p_{i-1} \cdot p_{i}$ can be derived if and only if $p_{i-1}$ has previously been received (by the direct link) and/or decoded successfully (from lower indices). We denote this probability of success as $\vec{P}^{(0)}$ and call it the forward decoding probability where

$$
\vec{P}_{i}^{(0)}=P_{\mathrm{RD}} \cdot\left(P_{\mathrm{SD}}+\left(1-P_{\mathrm{SD}}\right) \cdot \vec{P}_{i-1}^{(0)}\right) .
$$

- Finally, $p_{i}$ can be derived from a successful reception of an encoded packet which contains a combination of $p_{i} \oplus p_{i+1} . p_{i}$ can be decoded if and only if $p_{i+1}$ is already received and/or decoded successfully. We denote this probability of success for packet $i$ as $\overleftarrow{P}_{i}^{(0)}$ and call it the backward decoding probability where

$$
\overleftarrow{P}_{i}^{(0)}=P_{\mathrm{RD}} \cdot\left(P_{\mathrm{SD}}+\left(1-P_{\mathrm{SD}}\right) \cdot \overleftarrow{P}_{i+1}^{(0)}\right)
$$

As a result, the end-to-end success probability in sending each packet $p_{i}$ over a relay channel with network coding can be written as a double recursive expression:

$$
P_{i}^{(0)}=P_{\mathrm{SD}}+\left(1-P_{\mathrm{SD}}\right) \cdot\left(\vec{P}_{i}^{(0)}+\overleftarrow{P}_{i}^{(0)}-\vec{P}_{i}^{(0)} \overleftarrow{P}_{i}^{(0)}\right)
$$

with $\vec{P}_{0}^{(0)}=0$ and $\overleftarrow{P}_{N+1}^{(0)}=0$

Hence, when $N$ packets are to be transmitted over the relay channel, the average probability of success in the system is equal to:

$$
P_{N}^{(0)}=\frac{1}{N} \cdot \sum_{i=1}^{N} P_{i}^{(0)}
$$




\section{Realistic study : $P_{S R} \neq 1$}

We now consider the more realistic case where $P_{S R} \neq 1$. In this case, some of the packets sent by the source can be lost, and some of the previous combinations are not possible anymore. Indeed, if the buffer of the relay contains only one packet out of the 2 last ones sent by the source, the relay will send this packet. Furthermore, if the 2 last packets where lost, then no packet is sent by the relay.

This relaying process is described in Algorithm 1.

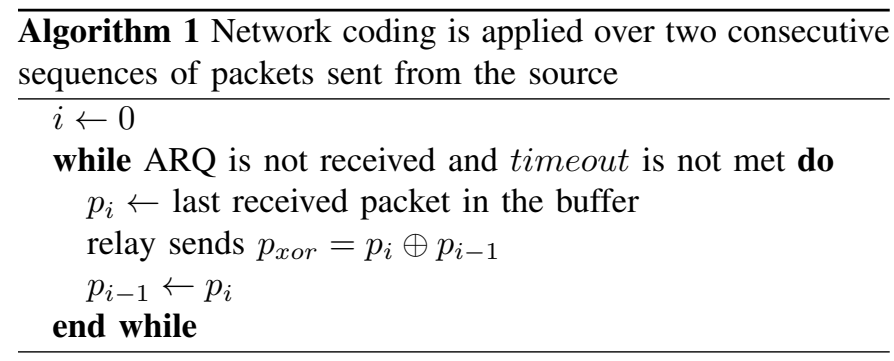

As a consequence, when the link S-R experiences losses, a given packet can be deducted from the forward and backward decoding if and only if it has been successfully transmitted over S-R. Thus, we introduce $P_{S R}$ into the previous expression (10) and we get:

$P_{i}^{(1)}=P_{\mathrm{SD}}+\left(1-P_{\mathrm{SD}}\right) P_{\mathrm{SR}}\left(\vec{P}_{i}^{(1)}+\overleftarrow{P}_{i}^{(1)}-\vec{P}_{i}^{(1)} \overleftarrow{P}_{i}^{(1)}\right)$

Besides, the probability based on the successful transmission and forward decoding is also impacted. Indeed, a packet which is at least containing $p_{i}$, to be consistant with (6) from the relay induces the decoding of $p_{i}$ iif

- the XOR packet is successfully received at destination

- AND the packet $p_{i-1}$ is cancelled

* either because it is received from the direct transmission $P_{\mathrm{SD}}$

* or because it is deduced from the forward decoding if $p_{i-1}$ was received at the relay

* or because $p_{i-1}$ was not received at the relay, and so, the XOR packet contains only $p_{i}$

Thus, the forward decoding probability can be written as:

$$
\vec{P}_{i}^{(1)}=P_{\mathrm{RD}}\left(P_{\mathrm{SD}}+\left(1-P_{\mathrm{SD}}\right)\left(P_{\mathrm{SR}} \vec{P}_{i-1}^{(1)}+\left(1-P_{\mathrm{SR}}\right)\right)\right)
$$

Similarly, the probability of success based on the backward decoding can be derived as:

$$
\overleftarrow{P}_{i}^{(1)}=P_{\mathrm{RD}}\left(P_{\mathrm{SD}}+\left(1-P_{\mathrm{SD}}\right)\left(P_{\mathrm{SR}} \overleftarrow{P}_{i+1}^{(1)}+\left(1-P_{\mathrm{SR}}\right)\right)\right)
$$

If we consider the transmission of $\mathrm{N}$ packets, the probability of success of the relay channel with this specific network coding scenario becomes:

$$
P_{N}^{(1)}=\frac{1}{N} \cdot \sum_{i=1}^{N} P_{i}^{(1)} .
$$

Although this relaying technique is simple and intuitive, it is not this practical since it is usually difficult and not useful to keep track of all the sequences of packets for the relay.
This is especially true if this scenario is applied to a more complex network that creates a number of new combinations of packets. We thus propose to study a more practical relaying algorithm which is scalable and can be implemented with little memory capacity at the relays.

Scenario 2: relay node applies network coding to any two consecutive packets from the relay point of view.

Instead of selecting the two packets to XOR together based on their sequence numbers as done previously, Scenario 2 combines the two lastly received packets upon packet reception. In other words, as a relay receives a packet, it selects the previously received packet in its buffer and combines it with a XOR operation. Formally:

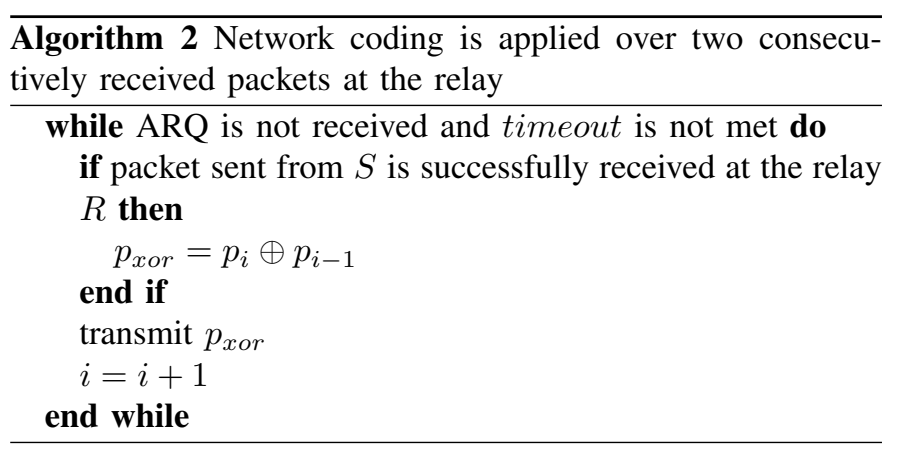

In the case of scenario 2, we can note that, from the destination point of view, the packets sent by the source can be divided into 2 distinct flows: the ones that succeed in reaching the relay, and the ones that don't. For the latter set of packets, the cooperative transmission acts as if they were directly transmitted to the destination without any intervention of the relay. Thus, for this set, the success probability is $P_{S D}$. For the remaining set of packets, the transmission acts as if they were perfectly received (so corresponding to the maximum diversity case $P_{S R}=1$ as in the preliminary study), coded and forwarded by the relay using Scenario 1 . Thus, their endto-end average success probability can be derived from the law of total probabilities:

$$
\begin{aligned}
P_{i}^{(2)} & =\left(1-P_{\mathrm{SR}}\right) \cdot P_{\mathrm{SD}}+P_{\mathrm{SR}} \cdot P_{i}^{(0)} \\
& =P_{\mathrm{SD}}+\left(1-P_{\mathrm{SD}}\right) \cdot P_{\mathrm{SR}} \cdot\left(\vec{P}_{i}^{(0)}+\overleftarrow{P}_{i}^{(0)}-\vec{P}_{i}^{(0)} \overleftarrow{P}_{i}^{(0)}\right)
\end{aligned}
$$

Again, if we consider the transmission of $N$ packets, the average success probability of the relay channel with this specific network coding scenario becomes:

$$
P_{N}^{(2)}=\frac{1}{N} \cdot \sum_{2=1}^{N} P_{i}^{(2)} .
$$

\section{Quasi-stationary Approximation}

In this section, we derive closed form approximations of equations (8) and (10) to avoid solving recursive equations which can lead to high computational cost as $N$ grows. To obtain the end-to-end success probability, we propose to observe the transmission in quasi-stationary state and we 
obtain, as $N \rightarrow \infty$, the end-to-end success probability with backward decoding from equation (6). For any $N \in \mathbb{N}$ :

$$
\begin{aligned}
\vec{P}_{N}^{(1)}= & \underbrace{P_{\mathrm{RD}}\left(1-P_{\mathrm{SD}}\right) P_{\mathrm{SR}}}_{\alpha} \vec{P}_{N-1}^{(1)} \\
& +\underbrace{P_{\mathrm{RD}} P_{\mathrm{SD}}+P_{\mathrm{RD}}\left(1-P_{\mathrm{SD}}\right)\left(1-P_{\mathrm{SR}}\right)}_{\beta}
\end{aligned}
$$

Knowing that $\vec{P}_{i}^{(1)}=\alpha \vec{P}_{i-1}^{(1)}+\beta$ is equal to $\vec{P}_{i}^{(1)}=\left(\vec{P}_{0}^{(1)}+\right.$ $\left.\frac{\beta}{\alpha-1}\right) \alpha^{i}-\frac{\beta}{\alpha-1}, \alpha \neq 1$, it can be rewritten as:

$$
\begin{aligned}
\vec{P}_{N}^{(1)}= & \frac{P_{\mathrm{RD}} P_{\mathrm{SD}}+P_{\mathrm{RD}}\left(1-P_{\mathrm{SD}}\right)\left(1-P_{\mathrm{SR}}\right)}{P_{\mathrm{RD}}\left(1-P_{\mathrm{SD}}\right) P_{\mathrm{SR}}-1} \\
& \times\left(\left(P_{\mathrm{RD}}\left(1-P_{\mathrm{SD}}\right) P_{\mathrm{SR}}\right)^{N}-1\right) .
\end{aligned}
$$

As $N \rightarrow \infty$, we have the symmetric property $P_{\mathrm{N} \rightarrow \infty}^{(1)}=$ $\vec{P}_{\mathrm{N} \rightarrow \infty}^{(1)}=\overleftarrow{P}_{\mathrm{N} \rightarrow \infty}^{(1)}$, we can simplify the equation (16) $\forall N \in$ $\mathbb{N}$ to:

$$
P_{\mathrm{N} \rightarrow \infty}^{(1)}=P_{\mathrm{SD}}+\left(1-P_{\mathrm{SD}}\right) P_{\mathrm{SR}}\left(2 P_{\mathrm{N} \rightarrow \infty}^{(1)}-P_{\mathrm{N} \rightarrow \infty}^{(1)}\right) .
$$

In the same manner, when the relay transmits a XOR combination of any two previously received packets, in a stationary regime, we can transform equation (2) for any $N \in \mathbb{N}$ :

$$
\vec{P}_{N}^{(2)}=\underbrace{P_{\mathrm{RD}}\left(1-P_{\mathrm{SD}}\right)}_{\alpha} \vec{P}_{N-1}+\underbrace{P_{\mathrm{RD}} P_{\mathrm{SD}}}_{\beta}
$$

into $\forall N \in \mathbb{N}$ :

$$
\vec{P}_{\mathrm{N} \rightarrow \infty}^{(2)}=\frac{P_{\mathrm{RD}} P_{\mathrm{SD}}}{1-P_{\mathrm{RD}}\left(1-P_{\mathrm{SD}}\right)}
$$

Having $N$ going to infinity, we get the equivalent success probability for the case where the relay forwards the two lastly received packets:

$$
\begin{aligned}
P_{\mathrm{N} \rightarrow \infty}^{(2)} & =P_{\mathrm{SR}} \cdot\left(P_{\mathrm{SD}}+\left(1-P_{\mathrm{SD}}\right)\left(2 P_{\mathrm{N} \rightarrow \infty}^{(2)}-P_{\mathrm{N} \rightarrow \infty}^{(2)}\right)\right) \\
& +\left(1-P_{\mathrm{SR}}\right) \cdot P_{\mathrm{SD}}
\end{aligned}
$$

\section{E. Simulation results}

This part focuses on the validation of our previously proposed theoretical analysis using simulation results. In our simulations, the source transmits a sequence of $N$ unencoded packets to the destination via a relay node. Transmission protocol follows the system model of Section III.

Figure 2 represents the end-to-end probability obtained for both network coding scenarios as a function of $P_{S R}$ and for several values of $P_{S D}$. Exact analytical values are obtained from equations (8) and (10). Simulation results perfectly match our derivations. The end-to-end success probability of Scenario 1 is higher than the one of Scenario 2: for any value of $P_{S D}<$ $1, P^{(1)}$ is higher than $P^{(2)}$. This result can be explained in the following way. Scenario 1 is the scenario where the relay forwards the most often packets. This is due to the fact that in the first scenario, an attempt to transmit the packet from the relay node is performed at each time slot whereas in the

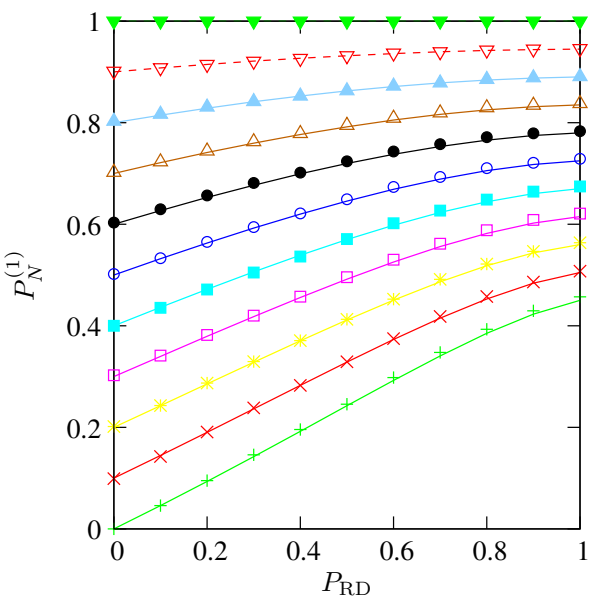

(a) Scenario 1

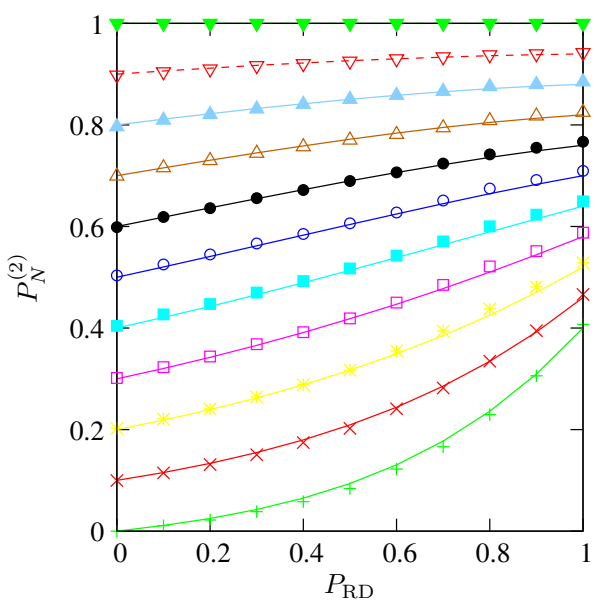

(b) Scenario 2

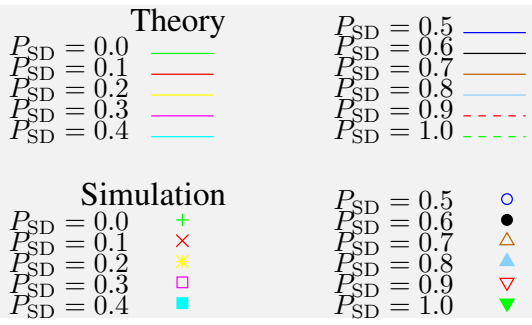

Fig. 2. Exact end-to-end success probability: analytical and simulation results for $N=10$ original packets.

second scenario, transmission at the relay node is launched if and only if the relay node receives a packet from the source. Thus, the probability for $D$ to receive an already decoded packet from $R$ is higher, which increases its chances to decode successfully previously received or upcoming network coded packets. In Scenario 2, all packets emitted by the relay are XOR-ed together. As such, the more non-combined packets get lost on the direct link from $S$ to $D$, the lower is the probability of $D$ to decode the original data.

Figure 3 compares the steady state approximation of the end-to-end success probability with exact derivations obtained 


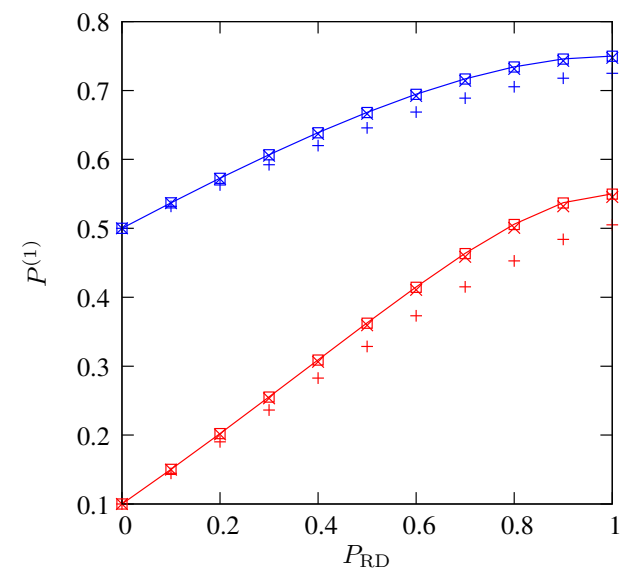

(a) Scenario 1

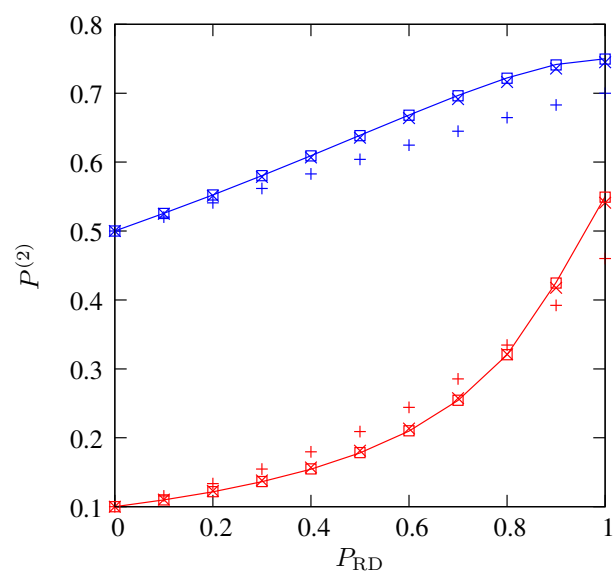

(b) Scenario 2

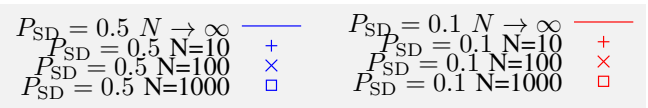

Fig. 3. End-to-end success probability: quasi-stationary approximation and exact derivation for $N \in\{10,100,1000\}$ and $P_{S D}=\{0.1,0.5\}$.

for $N \in\{10,100,1000\}$. As observed in figure 3 , we can see that the probability of success improves with the link quality on R-D and S-D. The quasi-stationary approximation gives a tight bound as the number of packets $N$ grows.

\section{TRANSMISSION RATE OPTIMIZATION}

When a relay node is introduced between the source and the destination, packet transmission can follow two paths to reach the destination. In fact, if the link probability between $\mathrm{S}-\mathrm{D}$ is perfect, transmission through an alternative path via the relay can cost unnecessary resource consumption. We propose in this section to find the equilibrium point where the relay benefits end-to-end communication without introducing too much redundancy. Therefore, we introduce $\tau$, the relay forwarding probability and look for the value that minimizes the average number of transmitted packets that are required to complete the transmission with $100 \%$ success rate.

\section{A. Theoretical Analysis}

We define here a new control parameter called the relaying rate and denoted $\tau \in[0,1]$. The equations (13) and (16) can be rewritten as a function of $\tau$. For the case where relay node applies network coding to any two consecutively transmit packets from the source, the overall probability of the system is equal to:

$$
\begin{aligned}
P_{\mathrm{N} \rightarrow \infty}^{(1)}= & P_{\mathrm{SD}}+\left(1-P_{\mathrm{SD}}\right) P_{\mathrm{SR}} \\
& {\left[2 \cdot \frac{\tau \cdot P_{\mathrm{RD}} P_{\mathrm{SD}}+\tau \cdot P_{\mathrm{RD}}\left(1-P_{\mathrm{SD}}\right)\left(1-P_{\mathrm{SR}}\right.}{1-\tau \cdot P_{\mathrm{RD}}\left(1-P_{\mathrm{SD}}\right) P_{\mathrm{SR}}}\right) } \\
& \left.-\left(\frac{\tau \cdot P_{\mathrm{RD}} P_{\mathrm{SD}}+\tau \cdot P_{\mathrm{RD}}\left(1-P_{\mathrm{SD}}\right)\left(1-P_{\mathrm{SR}}\right)}{1-\tau \cdot P_{\mathrm{RD}}\left(1-P_{\mathrm{SD}}\right) P_{\mathrm{SR}}}\right)^{(2)}\right]
\end{aligned}
$$

Otherwise, for the case where relay node applies network coding to any two consecutively received packets, the overall probability is equal to:

$$
\begin{aligned}
P_{\mathrm{N} \rightarrow \infty}^{(2)}= & P_{\mathrm{SR}} \cdot\left(P_{\mathrm{SD}}+\left(1-P_{\mathrm{SD}}\right)\left(2\left(\frac{\tau \cdot P_{\mathrm{RD}} P_{\mathrm{SD}}}{1-\tau \cdot P_{\mathrm{RD}}\left(1-P_{\mathrm{SD}}\right)}\right)\right.\right. \\
& \left.\left.-\left(\frac{\tau \cdot P_{\mathrm{RD}} P_{\mathrm{SD}}}{1-\tau \cdot P_{\mathrm{RD}}\left(1-P_{\mathrm{SD}}\right)}\right)^{(2)}\right)\right)+\left(1-P_{\mathrm{SR}}\right) \cdot P_{\mathrm{SD}} .
\end{aligned}
$$

The average number of transmissions $N_{t x}$ can still be written as a function of $P_{\mathrm{N} \rightarrow \infty}^{(1)}$ or $P_{\mathrm{N} \rightarrow \infty}^{(2)}$, both represented by variable $P_{e q}$, we obtain:

$$
N_{t x}=\frac{1}{P_{e q}} \cdot\left(1+P_{\mathrm{SR}} \tau\right)
$$

There is an optimal value for $N_{t x}$ which is controlled by the relaying rate $\tau$. Optimal $N_{t x}$ and corresponding relaying rates $\tau$ are plotted in Fig. 4 and 5. It can be seen that there is a minimum number of transmissions required to mitigate the loss on the channel. Based on the expression of the total number of transmissions which represents the system resource consumption, the equation (17) allows us to derive the optimum relaying rate which corresponds to the case where the overall number of transmissions becomes minimal $\left(\min \left(N_{t x}\right)\right)$.

Fig. 4 gives the results for $\min \left(N_{t x}\right)$ and its corresponding optimal $\tau$ as a function of $P_{S D}$ when $P_{S R}=P_{R D}=1$. We compare the results for the cases where the two network coding scenarios are applied, as well as the case where no network coding is applied. The results obtained confirm that when the quality of the relay link is perfect and when network coding is applied, the number of transmission required for successful transmission can be reduced by $10 \%$ and $25 \%$ respectively for the relaying scenarios 1 and 2 . However, when the relay link is degraded with $P_{S R}=P_{R D}=0.5$, as shown in Fig. 5 the scenario where we always transmit 2 consecutive packets from the source can incur an inefficiency at the decoder as there's no individual packet available at the decoder. This introduces an increase in the number of transmissions. We can conclude that the network coding in a relay channel is beneficial for 
the number of transmissions which lead to an improvement in resource consumption especially in the case where the direct link is highly degraded $\left(P_{S D} \lesssim 0.3\right)$

\section{Fountain Codes for a Relay Network}

As we have seen in the previous section, network coding brings higher efficiency in terms of the average number of transmissions in a cooperative transmission scheme. In this section, we are investigating the case where network coding is not applied on raw data, but on already encoded packets.

We are interested here in the case where transmitted data is subjected to two layers of encoding:

- the first layer consists of encoding RLF code at the source,

- the second layer consists of applying network coding at the relay. The relay re-encoded the received packets by applying linear combination in in $\mathbb{F}_{2}$ following the algorithm 1 and 2 .

We compare the transmission performance between the two scenarios. The first scenario represents the case where the relay node applies network coding to any two consecutive packets from the source point of view (algorithm 1) whereas the second scenario represents the case where network coding is applied to any two consecutive packets from the relay point of view (algorithm 2).

In Fig 6, the traffic overhead is presented as a function of $\tau$ when different relaying scenarios are considered. The overhead is defined as the number of packets transmitted by the source node divided by $N$, the number of original data packets. The link probability between each node is equal to $0.5\left(P_{S R}=\right.$ $\left.P_{R D}=P_{S D}=0.5\right)$. The overhead for the two investigated network coding scenarios and no coding case is computed by simulations. As presented in Fig. 6, network coding greatly reduces the overhead.

Contrary to the previous results where only network coding is performed (no RLF), when two layers coding is applied transmission performance of relaying with algorithm 2 (scenario 2) outperforms the one when algorithm 1 (scenario 1) is applied in term of traffic overhead. This is due to the fact that the algorithm 2 provided higher level of packet diversity. We has also observed that when the direct S-D link is bad $\left(P_{S D}=0.5\right)$, relay has to transmit at full rate to assist in the transmission. Fig. 6 clearly shows as well the benefits of the packet diversity introduced by network coding: the traffic overhead decreases as the relay forwards more and more packets.

\section{CONCLUSION}

In this paper, we have emphasized the benefits of network coding in a simple cooperative transmission scheme, the relay channel. We have developed a theoretical analysis of the endto-end success probability for two different network coding scenarios. Our theoretical analysis as well as the simulation results confirmed that network coding introduces gains in terms of energy and delay. The total number of transmission

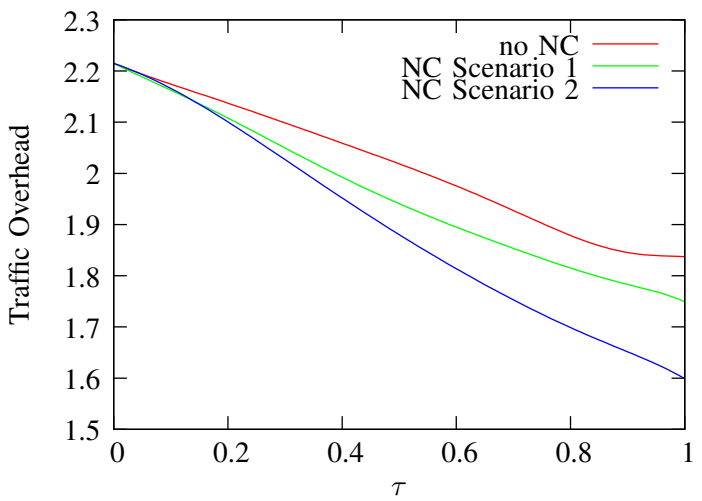

Fig. 6. Traffic overhead when network coding is applied at the relay $(N=$ $10)$.

can be reduced thanks to packet diversity introduced by effective network coding techniques.

To fully take advantage of this packet diversity, we have introduced a relaying decision variable to find the optimal point where the redundancy introduced by the relay is sufficient to combat error on the direct link without wasting energy with unnecessary transmissions. Finally, we have shown through simulations that the combination of an RLF code and a network code greatly improves the cooperative communication performance.

To conclude, we have demonstrated the benefits of network coding over a relay channel. This benefit can be obtained both in the case where the encoding is performed only at the relay or even in the case when the encoding process is performed both at the relay and the source.

\section{REFERENCES}

[1] R. Comroe and J. Costello, D., "Arq schemes for data transmission in mobile radio systems," Selected Areas in Communications, IEEE Journal on, vol. 2, no. 4, pp. 472 - 481, jul 1984.

[2] J. Lacan, V. Roca, J. Peltotalo, and S. Peltotalo, Reed-Solomon Forward Error Correction (FEC) Schemes, April 2009, iETF Request for Comments, RFC 5510 (Standards Track/Proposed Standard).

[3] R. Gallager, "Low-density parity-check codes," IRE Transactions on Information Theory, vol. 8, no. 1, pp. 21-28, January 1962.

[4] D. J. C. Mackay, "Fountain codes," IEE Communications, vol. 152, pp. 1062-1068, 2005.

[5] M. Luby, "LT Codes," in Proc. The 43rd IEEE Symposium on Foundations of Computer Science (FOCS), Vancouver, BC, Canada, November 2002, p. 271.

[6] A. Shokrollahi, "Raptor codes," IEEE Transactions on Information Theory, vol. 52, no. 6, pp. 2551-2567, 2006.

[7] J. N. Laneman, Cooperation in Wireless Networks: Principles and Applications, ser. Cooperative Diversity: Models, Algorithms, and Architectures. Springer, 2006.

[8] A. Apavatjrut, C. Goursaud, K. Jaffreè ands Runser, C. Comaniciu, and J. Gorce, "Toward increasing packet diversity for relaying lt fountain codes in wireless sensor networks," Communications Letters, IEEE, vol. 15 , no. 1 , pp. $52-54$, january 2011.

[9] S. Qin, G. Feng, and Y. Zhang, "Capacity bounds of cooperative communications with fountain codes," in Proc. IEEE Wireless Communications and Networking Conference (WCNC), April 2010, pp. 1-4.

[10] E. Kurniawan, S. Sumei, K. Yen, and K. Chong, "Network coded transmission of fountain codes over cooperative relay networks," in Wireless Communications and Networking Conference (WCNC), 2010 IEEE, April 2010, pp. 1-6. 

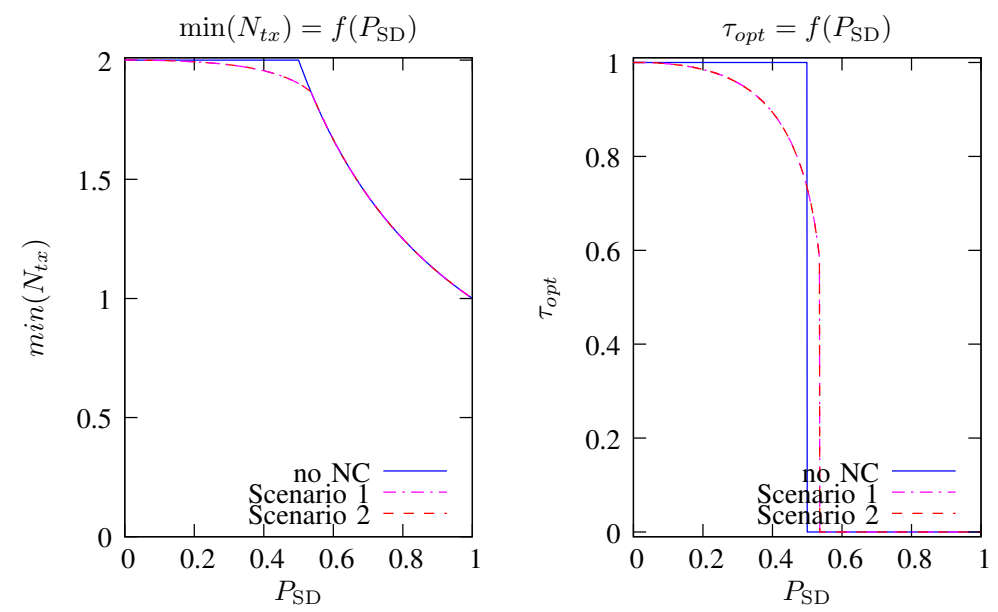

Fig. 4. Optimal number of transmissions vs. optimal relaying rate $\left(P_{\mathrm{SR}}=P_{\mathrm{RD}}=1\right)$
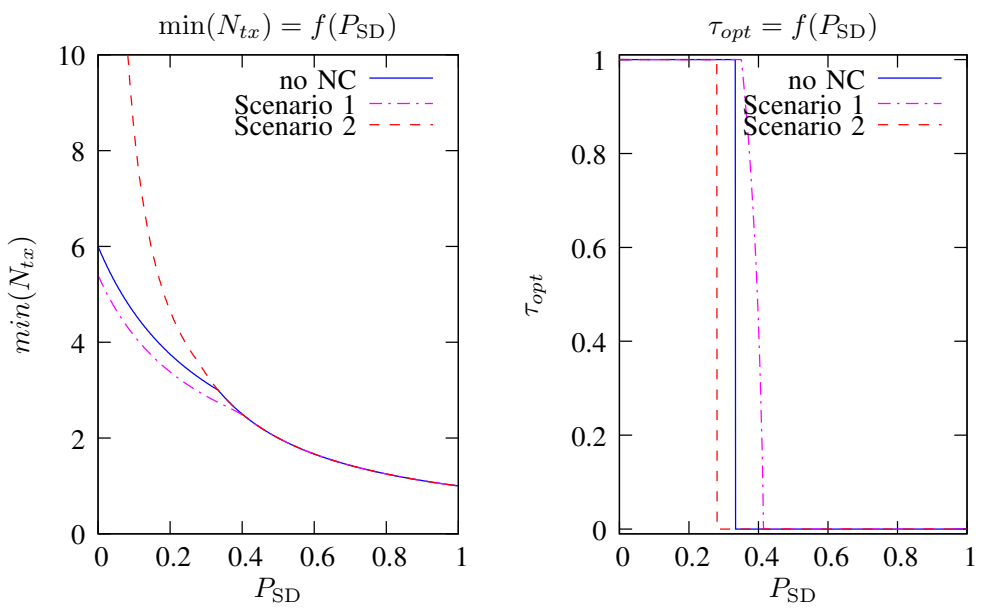

Fig. 5. Optimal number of transmissions vs. optimal relaying rate $\left(P_{\mathrm{SR}}=P^{(2)}=0.5\right)$

[11] J. W. Byers, M. Luby, M. Mitzenmacher, and A. Rege, "A Digital Fountain Approach to Reliable Distribution of Bulk Data," in Conference on Applications, Technologies, Architectures, and Protocols for Computer Communication (SIGCOMM), Vancouver, BC, Canada, 1998, pp. 56-67.

[12] A. Sendonaris, E. Erkip, and B. Aazhang, "User cooperation diversity. part i. system description," Communications, IEEE Transactions on, vol. 51, no. 11, pp. 1927 - 1938, nov. 2003.

[13] R. Ahlswede, N. Cai, S.-Y. Li, and R. Yeung, "Network information flow," IEEE Transactions on Information Theory, vol. 46, no. 4, pp. 1204-1216, July 2000.

[14] C. Fragouli, J. Widmer, and J.-Y. Le Boudec, "Efficient broadcasting using network coding," IEEE/ACM Transactions on Networking, vol. 16, no. 2, pp. 450-463, April 2008. 